\title{
EchoGéo
}

$57 \mid 2021$

Les enjeux de l'alimentation en eau potable des villes

\section{Reconquérir la qualité de l'eau potable par le développement de l'agriculture biologique et de systèmes alimentaires dédiés}

Le cas de la vallée de la Vanne et de la ville de Paris

\section{Audrey Vincent et Philippe Fleury}

\section{OpenEdition}

Journals

Édition électronique

URL : https://journals.openedition.org/echogeo/22457

DOI : $10.4000 /$ echogeo.22457

ISSN : 1963-1197

Éditeur

Pôle de recherche pour l'organisation et la diffusion de l'information géographique (CNRS UMR 8586)

Référence électronique

Audrey Vincent et Philippe Fleury, «Reconquérir la qualité de l'eau potable par le développement de l'agriculture biologique et de systèmes alimentaires dédiés », EchoGéo [En ligne], 57 | 2021, mis en ligne le 15 octobre 2021, consulté le 22 novembre 2021. URL : http://journals.openedition.org/ echogeo/22457; DOI : https://doi.org/10.4000/echogeo.22457

Ce document a été généré automatiquement le 22 novembre 2021.

EchoGéo est mis à disposition selon les termes de la licence Creative Commons Attribution - Pas d'Utilisation Commerciale - Pas de Modification 4.0 International (CC BY-NC-ND) 


\section{Reconquérir la qualité de l'eau potable par le développement de l'agriculture biologique et de systèmes alimentaires dédiés}

Le cas de la vallée de la Vanne et de la ville de Paris

Audrey Vincent et Philippe Fleury

Remerciements. Le travail présenté dans cet article a été réalisé dans le cadre d'un projet de recherche ayant bénéficié de l'appui de l'agence de l'eau Rhône Méditerranée Corse.

\section{Introduction}

\section{L'alimentation en eau potable : un enjeu environnemental et de santé publique}

1 L'alimentation en eau potable est un enjeu majeur pour les collectivités locales en France. En effet celles-ci ont l'obligation de distribuer aux citoyens une eau respectant les normes de potabilité. Pour être considérée comme « potable », l'« eau du robinet » doit être conforme à certaines normes relatives à des paramètres micro-biologiques, physiques et chimiques fixées par la Directive Eau Potable publiée en 1980. Le nonrespect de ces normes peut conduire à des abandons de captages ou à un nécessaire traitement de potabilisation de l'eau avant sa distribution.

2 La Directive européenne Cadre sur l'Eau, publiée en 2000, fixe un objectif de bon état des masses d'eau et milieux aquatiques. L'échéance, initialement fixée pour 2015, a été échelonnée jusqu'en 2021 et 2027. La reconquête de la qualité des ressources destinées à l'alimentation en eau potable est donc un enjeu stratégique, environnemental comme de santé publique. 


\section{L'agriculture biologique comme solution face aux problèmes de pollutions diffuses agricoles}

3 Pourtant, de nombreux captages rencontrent des problèmes de pollution, notamment par les nitrates et les pesticides utilisés en agriculture. Ces pollutions diffuses agricoles persistent en dépit des actions mises en place au cours des dernières décennies. Face à ce constat, le Grenelle de l'environnement en 2007 a mis en avant la nécessité d'actions préventives visant à engager des changements de fonds notamment dans les pratiques agricoles. L'agriculture biologique $(\mathrm{AB})$, de par ses principes, ses pratiques et son règlement, est apparue comme une possible réponse (Fleury, 2011 ; Thieu et al., 2011). La loi Grenelle 1, publiée en 2009, a ainsi mis l'accent sur le rôle que pourrait jouer l'AB dans la préservation des ressources en eau et a fixé un objectif de développement de l'AB dans les aires d'alimentation de captages (AAC) ${ }^{1}$. L'AAC est définie sur des bases hydrogéologique comme étant la surface sur laquelle toute goutte d'eau tombée au sol est susceptible de parvenir à l'ouvrage de captage. Elle est considérée comme l'échelle d'action pertinente à privilégier pour reconquérir la qualité des ressources en eau destinées à « l'alimentation en eau potable ».

Cet objectif de développement de l'AB pour reconquérir la qualité des ressources destinées à l'alimentation humaine a directement interpellé les collectivités gestionnaires de captages. Suite au Grenelle de l'Environnement, une liste de 500 captages considérés comme "prioritaires » a été publiée ; cette liste a depuis été portée à 1000 captages lors de la Conférence Environnementale de 2013. Les collectivités gestionnaires de captages devaient y mettre en place un programme d'action pour reconquérir la qualité de l'eau.

\section{Enjeux et question de recherche}

Différents travaux montrent que la mise en œuvre de l'objectif de la loi Grenelle 1 visant à favoriser le développement de l'AB dans les $A A C$ est complexe et reste limité (Barataud et al., 2014; Epices et ADE, 2017, Epices et AScA, 2020). En effet, le passage à l'AB est un changement de fond pouvant nécessiter une reconception en profondeur des systèmes de production (Bellon et Lamine, 2009). Beaucoup d'agriculteurs hésitent à s'engager dans un changement d'une telle ampleur et préfèrent privilégier de simples ajustements de leurs pratiques comme une réduction limitée de l'usage des pesticides et des engrais de synthèse ou le remplacement de certains produits par d'autres considérés comme moins toxiques (Allaire et al., 2013; Epices et AScA, 2020). Ainsi, malgré les objectifs affichés, une analyse intermédiaire des actions mises en œuvre sur les captages prioritaires Grenelle a mis en évidence en 2014 que sur 137 captages Grenelle, l'AB apparait seulement dans 30\% des programmes d'action (Barataud et al., 2014). Un travail plus récent d'évaluation des mesures mises en place sur les territoires à enjeu eau a démontré que les dynamiques et niveaux de développement de l'AB y sont en général plus faibles que sur le reste du territoire national (Epices et $A D E, 2017$ ). Ce même rapport précise que, sur un échantillon de 75 captages, l'AB est citée dans $90 \%$ des programmes d'actions mais la référence à l'AB est dans la plupart des cas « un volet parmi d'autres » (Epices et ADE, 2017). L'AB est essentiellement présentée comme une solution optionnelle, qui sera mobilisée "si possible", tandis que le cœur de la démarche de reconquête de la qualité de l'eau repose sur des actions d'amélioration des 
pratiques agricoles conventionnelles, par exemple en réduisant la fréquence d'utilisation des pesticides.

6 Quelques cas font cependant exception et des dynamiques notables de développement de l'AB y sont observées. L'AB est alors posée comme un axe central du projet de reconquête de la qualité de l'eau (Epices, 2016 ; Epices et ADE, 2017). La bibliographie montre que deux difficultés semblent se cumuler dans le choix de cette voie. La première tient à la nature même du changement à engager par les agriculteurs. Le passage à l'AB suppose des transformations qui dépassent le seul aspect technique et l'échelle de la parcelle agricole mais qui concernent l'ensemble de l'exploitation, les circuits de commercialisation (Lamine et al., 2009), les réseaux d'échanges voire les conceptions du métier et les valeurs des agriculteurs (Bellon et Lamine, 2009). La conversion à l'AB relève donc d'un changement de fonds des systèmes.

7 La seconde difficulté réside dans la nécessité d'aller vers un développement territorialisé de l'AB. Pour espérer avoir un impact sur la qualité de l'eau au captage il faut en effet, qu'une part importante de la surface agricole soit en $A B$ (Vincent et Fleury, 2015). Il n'est pas possible de chiffrer ceci de façon absolue, mais ce pourcentage en $A B$ doit être largement supérieur à la moyenne nationale de la surface agricole en $A B$ qui est actuellement de 8,5\%. Il s'agit donc de cibler un développement important de l'AB dans les limites d'un territoire de gestion de l'eau, celui de l'AAC.

8 Cet objectif de développement territorialisé constitue un changement important par rapport à la façon dont le développement de l'AB est pensé classiquement en s'appuyant sur l'accompagnement d'agriculteurs motivés et sur l'appui à la structuration de filières de mise en marché des produits (Vincent, 2016). Or le territoire de l'AAC est déterminé sur des critères hydrogéologiques et ne coïncide pas, ou très rarement, avec les territoires d'action des acteurs agricoles: territoires des exploitations agricoles, bassins de collecte d'une coopérative agricole ou d'une entreprise, etc. (Petit et al., 2016). Les conditions favorables et les actions à entreprendre pour inciter à un développement territorialisé de l'AB dans les AAC sont méconnues et constituent un enjeu de recherche important.

9 En référence à ces deux difficultés, dans cet article, nous abordons la problématique suivante : comment une collectivité territoriale peut-elle concevoir et accompagner sur le temps long un projet de développement territorialisé de l'AB à l'échelle d'une AAC pour garantir la pérennité de son approvisionnement en eau potable? Nous cherchons en particulier à analyser quels outils d'action publique peuvent être mobilisés par une collectivité gestionnaire de captages pour favoriser le développement de l'AB à l'échelle d'une AAC. Pour cela, nous nous appuyons sur un cas original, celui de la ville de Paris et des actions de développement de l'AB mené par sa régie « Eau de Paris » sur l'AAC de la vallée de la Vanne (Yonne/Aube) dont les captages sont classés prioritaires Grenelle. Eau de Paris est en charge de la production, du transport et de la distribution en eau potable pour Paris. Afin de reconquérir la qualité de l'eau des captages de la vallée de la Vanne, cet opérateur public a structuré depuis 2008 un programme d'actions fondé exclusivement sur le développement de l'AB. Nous cherchons à analyser la structuration du projet, les actions et outils sur lesquels il repose et son évolution au cours du temps. 


\section{Cas de la vallée de la Vanne et méthodologie de recherche}

La ville de Paris est alimentée en eau potable par des prélèvements réalisés pour une moitié dans des ressources en eau superficielles (issues de la Seine et de la Marne) et pour l'autre moitié dans des ressources souterraines captées en zones rurales, jusqu'à $150 \mathrm{~km}$ de la capitale. Ces captages en eaux souterraines résultent d'ouvrages, réalisés entre la fin du XIXe et le début du XXe siècle, dans trois aires d'alimentation de captages principales : celle des sources de la Voulzie (Seine et Marne), de la Vigne (Eure et Loir) et de la vallée de Vanne (Yonne et Aube) (Zakeossian, 2011).

11 L'AAC de la vallée de la Vanne est située à une centaine de kilomètres au sud-est de Paris (illustration 1). Elle s'étend sur 41 communes des départements de l'Yonne et de l'Aube (illustration 2). Sur les 45000 ha de l'AAC dont 30000 ha de Surface Agricole Utile (SAU) environ, l'activité agricole est largement dominée par les grandes cultures. Certaines exploitations ont conservé un atelier d'élevage ou développé des productions de légumes, en plein champ ou sous serre mais elles sont minoritaires et les surfaces concernées sont faibles. Dans ce paysage de grandes cultures, certains éléments comme les ouvrages de pompage ou les aqueducs destinés à transporter l'eau par gravité jusqu'à Paris (illustration 3) rappellent ponctuellement l'enjeu d'approvisionnement en eau de la capitale.

Illustration 1 - Localisation de l'AAC de la vallée de la Vanne à une centaine de kilomètres au Sud Est de Paris

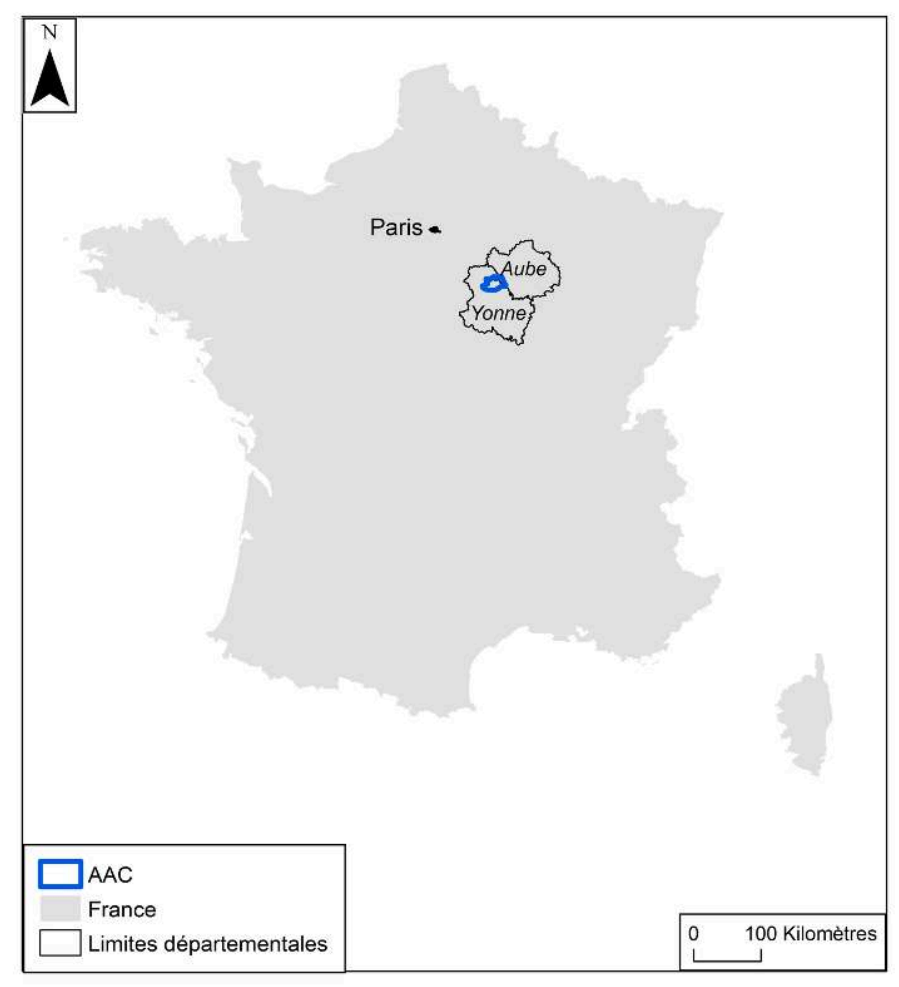

Source : IGN - Réalisation : A. Vincent. 
Illustration 2 - Aire d'alimentation de la vallée de la Vanne s'étendant sur les départements de l'Aube et de l'Yonne

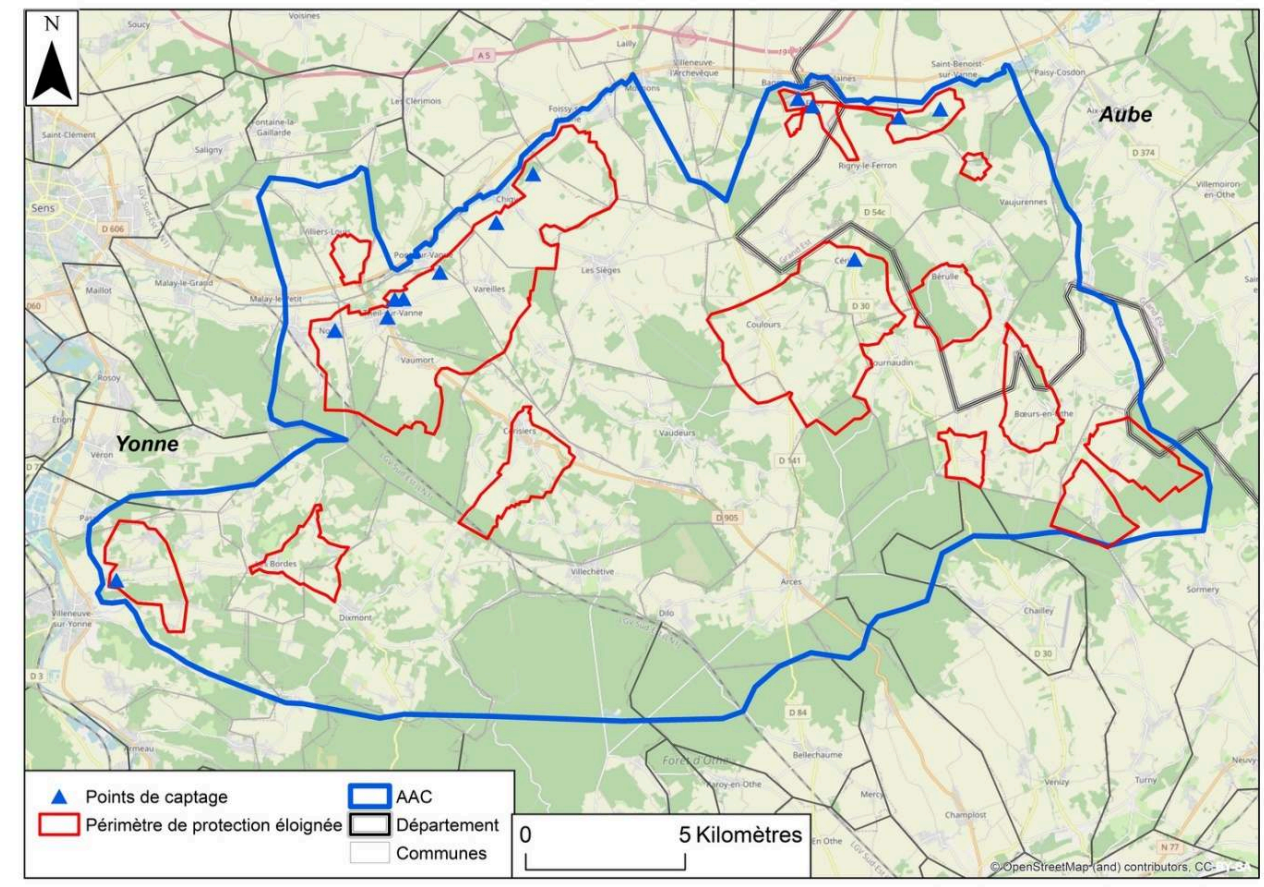

Source : IGN, OpenStreetMap 2021 - Réalisation : A. Vincent.

En 2008, les problèmes de pollution par les pesticides et les nitrates ont amené Eau de Paris à construire une unité de traitement de l'eau issue de la vallée de la Vanne (notamment pour le traitement des contaminations par les pesticides avant distribution). En parallèle, Eau de Paris a décidé de mener des actions de reconquête de la qualité de l'eau au sein de l'AAC et de baser ce projet exclusivement sur le développement de l'AB. Ce choix a été fait pour l'AAC de la vallée de Vanne uniquement. Il n'a pas été décliné sur les autres AAC de la ville de Paris qui sont situées dans des zones ayant un potentiel de production agricole supérieur et où la profession agricole semblait plus réticente à ce mode de production. Le choix de centrer les actions de reconquête de la qualité de l'eau sur le développement de l'AB est avant tout motivé par un souci d'efficacité. La personne responsable du service protection de la ressource explique ainsi que l'AB leur apparaissait comme la seule solution véritablement pérenne. En effet, ce changement, pensé à l'échelle de l'exploitation, peut perdurer dans le temps si l'agriculteur réussit à trouver un débouché rémunérateur pour ses productions biologiques. Selon elle, les solutions intermédiaires relevant de l'ajustement de pratiques (réduction de la fertilisation ou des pesticides) expérimentées sur les autres AAC de la capitale (Vigne et Voulzie) depuis les années 1990 se sont révélées insuffisantes. Le développement de l'AB est aussi une solution qui séduit alors les élus de la ville de Paris et notamment l'adjointe au maire en charge de l'eau qui préside également la régie. 
Illustration 3 - Aqueduc servant au transport de l'eau vers Paris

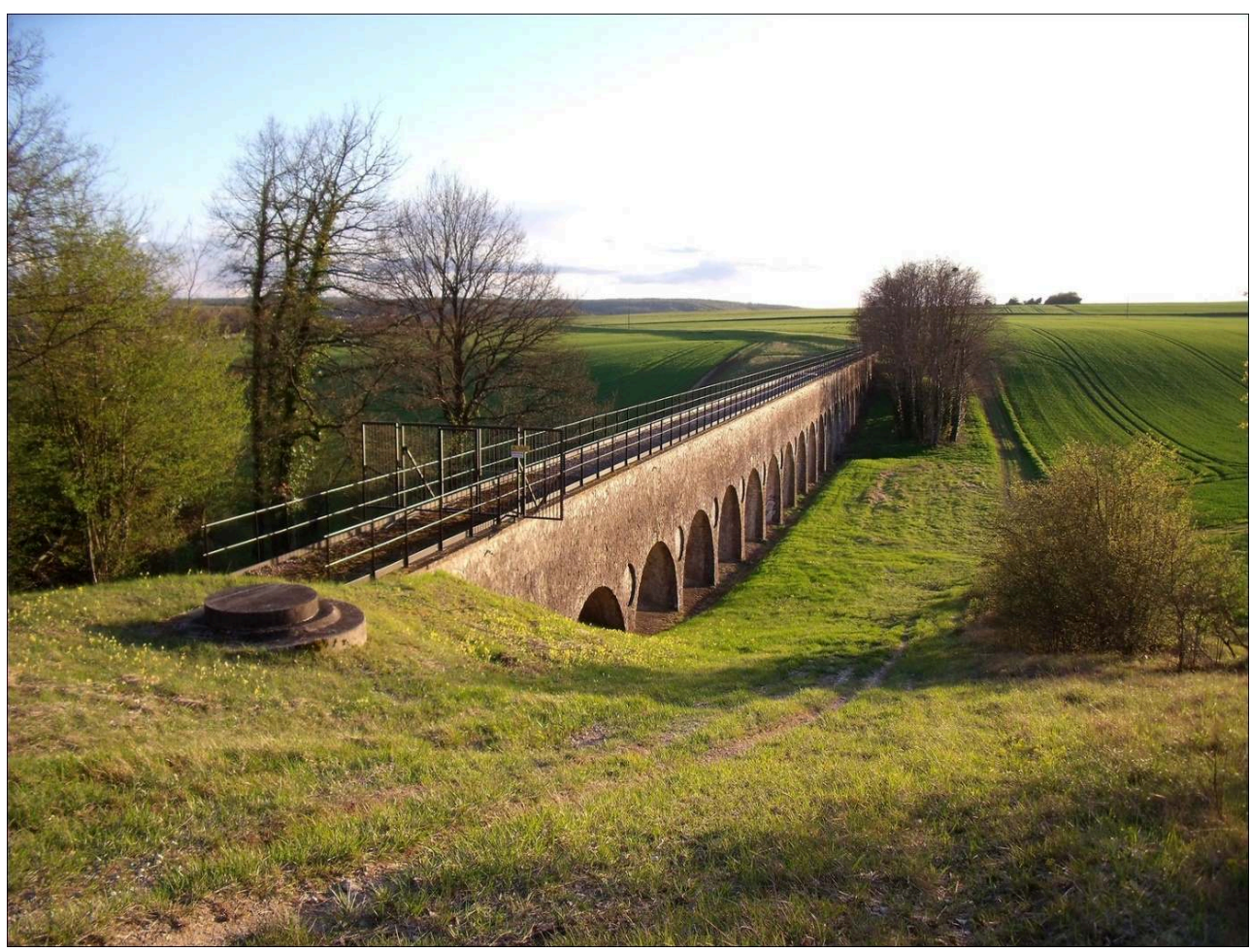

AAC de la vallée de la Vanne au niveau de la commune de Pont sur Vanne.

Auteur: A. Vincent.

13 Notre travail s'appuie sur la réalisation de 36 entretiens semi-directifs avec une diversité d'acteurs impliqués (gestionnaire de captages de la ville de Paris, agriculteurs biologiques et conventionnels ayant des parcelles sur l'AAC, animateurs locaux, opérateurs économiques assurant la collecte et mise en marché des produits agricoles, etc). Ces entretiens ont été réalisés en 2012, 2017 et 2021, en présentiel ou par téléphone auprès des principaux types d'acteurs impliqués dans le projet (Tableau 1).

Tableau 1 - Entretiens réalisés au cours des trois séries d'enquête

\begin{tabular}{|l|l|l|l|l|}
\hline $\begin{array}{l}\text { Gestionnaire de } \\
\text { captages }\end{array}$ & Agriculteurs & $\begin{array}{l}\text { Acteurs du } \\
\text { développement agricole }\end{array}$ & $\begin{array}{l}\text { Opérateurs de la collecte et } \\
\text { mise en marché }\end{array}$ \\
\hline 2012 & 1 & 17 & 2 & 5 \\
\hline 2017 & 1 & 2 & 1 & 1 \\
\hline 2021 & 1 & 2 & 1 & 2 \\
\hline
\end{tabular}

14 Les trois séries successives d'entretiens donnent une vision sur un pas de temps long et permettent de saisir les évolutions du projet en relation avec des changements des contextes règlementaire, sociétal et d'action publique. Cette approche diachronique de l'action publique «en train de se faire» (Musselin, 2005) permet d'appréhender les modalités de mise en œuvre du projet comme les ajustements ou les réorientations opérés pour le relancer ou le réorienter. Les premiers entretiens ont porté sur la 
description du projet, ses conditions d'élaboration, les actions mises en œuvre, sur les raisons ayant amené les différents acteurs à s'y engager, sur leur vision des problèmes de pollution rencontrés localement et de l'AB. Les suivants ont concerné l'évolution du projet, des outils d'action publique mobilisés et des conditions de mise en œuvre.

Par ailleurs, une analyse des documents relatifs au projet a été réalisée : coupures de presse, rapports d'activités, sites internet, brochures d'information ou de communication, dossiers de financement, etc.

16 Afin de mieux comprendre pourquoi et comment différents outils d'action publique ont été mobilisés pour accompagner le développement territorialisé de l'AB, nous avons veillé dans l'analyse des entretiens et des documents à bien rendre compte du raisonnement stratégique du gestionnaire, à ses stratégies d'alliance et de relations avec les acteurs agricoles locaux, aux controverses qui ont émergé comme aux arrangements qui se sont construits avec ces mêmes acteurs (Musselin, 2005).

\section{Résultats. Les étapes du projet de reconquête de la qualité de l'eau par le développement de l'AB sur l'AAC}

Nous présentons dans cette partie la trajectoire du projet de reconquête de la qualité de l'eau porté par Eau de Paris sur l'AAC de la vallée de la Vanne (illustration 4). L'analyse réalisée permet de faire apparaitre les différentes phases du projet qui correspondent à la mise en œuvre de nouveaux outils d'action publique et reflètent une évolution de la stratégie d'intervention du gestionnaire de captages.

\section{Une action centrée à l'origine sur l'AAC et l'animation agricole territoriale}

Dès 2008, Eau de Paris décide de lancer un projet de reconquête de la qualité de l'eau sur la vallée de la Vanne pour faire face aux problèmes de pollutions. Le choix est de baser ce projet sur le développement de l'AB pour engendrer des changements de fond des pratiques et systèmes agricoles. Il s'agit d'une démarche engagée à l'initiative du gestionnaire, avant que les captages ne soient classés prioritaires Grenelle.

Le gestionnaire de l'eau engage alors des discussions avec les acteurs du développement agricole afin de présenter son projet. La chambre d'agriculture exprime son désaccord quant à l'orientation envisagée et l'invite à revoir ses ambitions. Le groupement régional d'agriculture biologique (SEDARB) se dit à l'inverse être prêt à s'impliquer. Un partenariat est alors initié entre Eau de Paris et le SEDARB pour assurer une animation territoriale visant à sensibiliser les agriculteurs à l'enjeu " eau » et aux objectifs de développement de l'AB du gestionnaire de captages et à accompagner les agriculteurs intéressés par une conversion à l'AB. Eau de Paris finance ainsi, depuis 2008, un poste d'animation au SEDARB, devenu BioBourgogne, complètement dédié au développement de l'AB sur l'AAC de la vallée de la Vanne.

Le gestionnaire de captages associe ensuite d'autres outils à cette animation agricole territoriale. A partir de 2010, une MAET « conversion à l'agriculture biologique » est ouverte sur le territoire de l'AAC, permettant d'allouer un soutien financier d'un montant supérieur à celui d'une aide à la conversion « classique ». Il s'agit pour Eau de Paris d'utiliser les possibilités qu'offre le programme de développement rural de la 
politique agricole commune (PAC) pour apporter un soutien financier incitatif aux agriculteurs qui accepteraient de s'engager en $\mathrm{AB}$. Un partenariat ancien, datant de 1997, entre le gestionnaire de l'eau et la SAFER est également réactivé afin de renforcer la veille foncière à l'échelle de toute l'AAC. L'objectif est de réaliser des acquisitions foncières et de remettre les parcelles acquises à disposition d'agriculteurs s'engageant à les cultiver en $\mathrm{AB}$ (ou à les remettre en herbe), via des baux environnementaux.

\section{Un appui à la structuration de filières et à la recherche de débouchés rémunérateurs}

21 En 2011, un partenariat est mis en place avec les opérateurs économiques de la collecte de productions agricoles biologiques intervenant sur l'AAC. Cette collaboration prend d'abord une forme simple: les opérateurs économiques sont associés aux réunions d'information et de sensibilisation des agriculteurs afin de présenter les débouchés existants pour les productions biologiques. Fin 2011, Eau de Paris s'engage dans une action plus ambitieuse en soutenant financièrement un projet porté par deux coopératives biologiques et visant à créer une station de production de semences biologiques. L'objectif est de s'impliquer dans la structuration de débouchés nouveaux et à forte valeur ajoutée. Dans le même temps, Eau de Paris engage une réflexion avec la ville de Paris pour étudier les possibilités de valoriser les productions biologiques issues de l'AAC dans la restauration collective de l'agglomération. Cette idée sera ensuite temporairement abandonnée en raison de la rigidité des cadres imposés par le code des marchés publics.

Eau de Paris renforce également sa collaboration avec la coopérative céréalière $100 \%$ biologique en cofinançant un poste de conseiller technique. Complémentaire à l'animation territoriale déjà en place, ce poste vise un accompagnement technique et stratégique intégrant des enjeux relatifs aux débouchés ou aux attentes du marché, pour des agriculteurs en $\mathrm{AB}$ ou en conversion. Ouvrir ce poste au sein d'une coopérative agricole est vu comme un moyen de toucher un public élargi d'agriculteurs, certains pouvant être plus attentifs au conseil technique délivré par un opérateur économique.

\section{La structuration de collectifs d'agriculteurs}

En 2015, les échanges avec les agriculteurs biologiques locaux amènent le gestionnaire de captages à impulser la construction d'un collectif afin de «structurer les conversions et les pérenniser ». Pour lui, la mise en place d'un collectif était le moyen de créer du lien entre agriculteurs et ainsi d'éviter que des agriculteurs, isolés et démunis face à certaines impasses techniques ou économiques ne retournent vers l'agriculture conventionnelle. L'association Agribio Vanne et Othe est créée en juin 2015, puis reconnue comme GIEE (Groupement d'Intérêt Economique et Environnemental ${ }^{2}$ ), en décembre 2015. Elle regroupe alors 17 agriculteurs ${ }^{3}$, ayant tous au moins une parcelle en $A B$ sur l'AAC de la vallée de la Vanne. L'animation est assurée par le groupement d'agriculteurs biologiques de Bourgogne, avec le soutien financier d'Eau de Paris et de l'agence de l'eau Seine Normandie. L'agriculteur qui préside l'association explique qu'elle permet d'avoir un lieu d'échanges entre agriculteurs sur des enjeux techniques, économiques, de débouchés et d'évolution des exploitations (implantation de nouvelles cultures, diversification etc.). Elle constitue aussi un lieu 
d'échanges entre agriculteurs et gestionnaires de l'eau puisqu'Eau de Paris mais également un syndicat local de gestion de l'eau potable participent à l'assemblée générale.

Les discussions entre les agriculteurs de l'association les conduisent à réfléchir aux moyens d'améliorer leurs résultats économiques via un travail sur les marges brutes par culture. Une réflexion sur l'opportunité d'investissements en commun pour des achats de matériels émerge. L'objet de l'association ne permettant pas de concrétiser ces investissements, une CUMA (coopérative d'utilisation de matériel agricole) bio est créée en 2016 pour l'achat collectif d'un premier matériel. L'association est également un lieu de discussion sur les possibilités de diversification des exploitations, pour des raisons économiques mais aussi agronomiques de diversification des assolements et d'allongements des rotations. Un groupe d'agriculteurs engage par exemple une réflexion sur l'introduction de la culture du chanvre. Cette culture, peu consommatrice en intrants, est intéressante pour la préservation de la qualité de l'eau et pour les agriculteurs. Le marché du chènevis est en plein développement et constitue un débouché prometteur. La CUMA est alors mobilisée pour acheter en commun le matériel de récolte de cette nouvelle culture et permettre ainsi son implantation chez les agriculteurs intéressés.

\section{Associer eau de qualité et alimentation biologique dans la restauration collective}

Les discussions au sein de l'association AgriBio Vanne et Othe ont fait émerger la volonté de certains agriculteurs de s'organiser pour assurer eux-mêmes la mise en marché de certaines productions comme les lentilles. Cela a permis de relancer les discussions sur l'approvisionnement de cantines de la capitale avec des produits issus de l'AAC. Cette volonté coïncide alors également avec l'objectif du Plan Alimentation Durable 2015-2020 de la ville de Paris d'atteindre $50 \%$ d'alimentation "durable " (produits biologiques, label rouge...) dans les cantines d'ici à 2020. Eau de Paris assure la mise en lien des élus parisiens et des agriculteurs de l'AAC. La présidente du conseil d'administration d'Eau de Paris est alors l'adjointe à la mairie de Paris chargée de l'Environnement et du Développement Durable. En 2017, quelques agriculteurs réalisent les premières livraisons de lentilles issues de l'AAC pour les cantines du $11^{\text {ème }}$ arrondissement de Paris. Les contraintes imposées par le code des marchés publics incitent les agriculteurs à se structurer pour répondre collectivement aux appels d'offres de la ville de Paris.

En 2018, cinq agriculteurs créent l'association Terres du Pays d'Othe pour travailler à la commercialisation en restauration collective et en local de produits agricoles de l'AAC. Deux agriculteurs sont aujourd'hui impliqués dans l'association qui assure le conditionnement et la mise en marché de différents produits issus de l'AAC (lentilles, pois chiches, farines, différentes huiles, pâtes, etc.). Les produits, issus de différentes fermes de l'AAC, sont ainsi vendus de manière centralisée sous la marque «Terres du Pays d'Othe " dans des cantines de Paris, notamment dans les $11^{\text {ème }}$ et $12^{\text {ème }}$ arrondissements. 


\section{Un régime d'aides directes aux agriculteurs biologiques ou en conversion}

Enfin, Eau de Paris travaille avec les agriculteurs à l'élaboration d'un système d'aides qui soit mieux adapté aux besoins et spécificités rencontrés sur ses AAC et plus pérenne que les aides permises par le plan de développement rural de la PAC mobilisé jusqu'alors. En 2019, un dossier de notification est déposé à la Commission européenne. Il est validé en 2020, permettant à Eau de Paris de créer son propre régime d'aides aux agriculteurs. Ces aides fonctionnent sur le principe des paiements pour services environnementaux. Sur la vallée de la Vanne, Eau de Paris propose une seule mesure qui vise à accompagner les exploitations en $\mathrm{AB}$ ou en conversion. Basée sur un contrat de 7 ans, cette aide financière permet d'accompagner les agriculteurs jusqu'à la $15^{\text {ème }}$ année suivant la conversion en distinguant trois stades d'accompagnement: en conversion, en transition ou en maintien. Elle permet ainsi de pallier la suppression par le ministère, au niveau national, de l'aide au maintien en $A B$. Les aides versées aux agriculteurs sont financées par Eau de Paris, pour $20 \%$ et par l'agence de l'eau Seine Normandie pour $80 \%$.

Illustration 4 - Historique de la démarche engagée par Eau de Paris pour le développement de l'AB sur l'AAC de la vallée de la Vanne

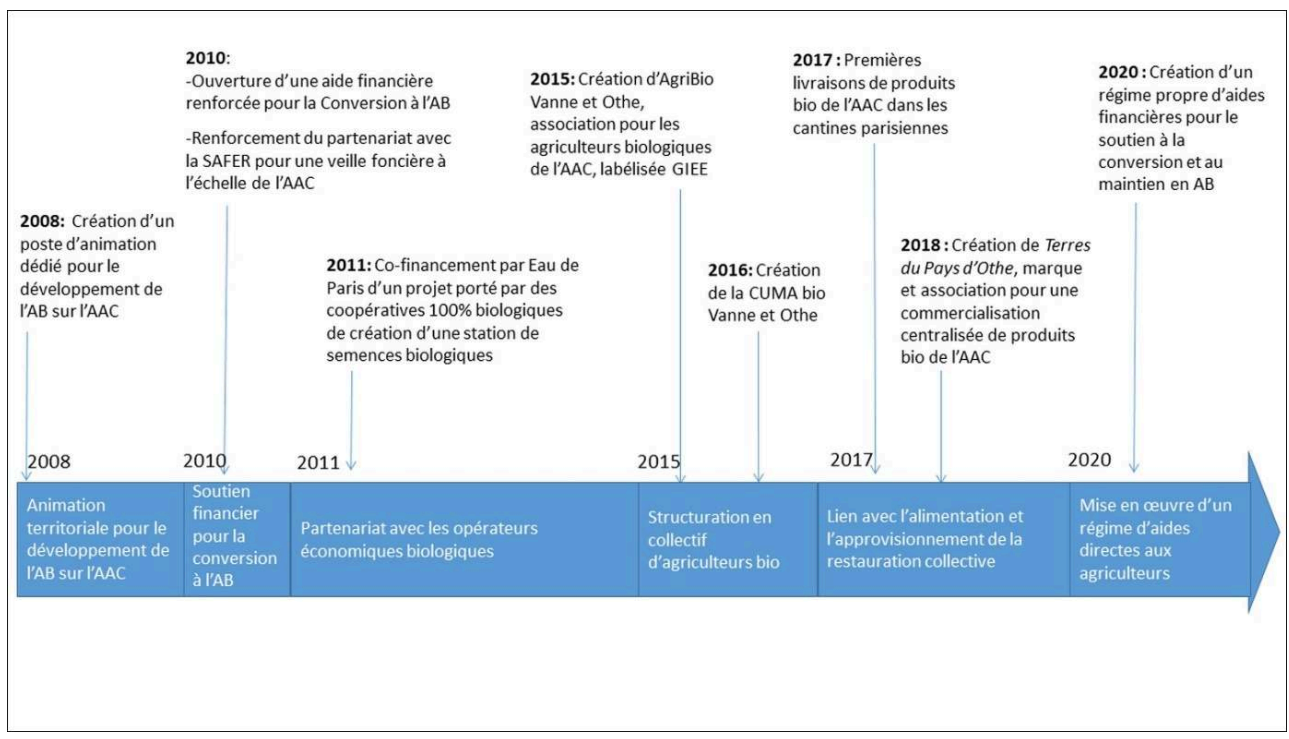

Réalisation : A. Vincent ; d'après Vincent, 2016.

\section{Discussion. Un projet de reconquête de la qualité de l'eau reposant sur l'articulation de politiques, d'outils et d'échelles d'actions}

\section{Articulation de différents outils d'action publique et d'échelles d'intervention}

L'historique du projet de reconquête de la qualité de l'eau mené sur la vallée de la Vanne met en évidence que celui-ci s'est complexifié au cours du temps pour répondre 
aux spécificités locales et aux besoins des agriculteurs. Cette complexification passe à la fois par le recours à un nombre croissant, à une diversité accrue d'outils d'action publique et par l'articulation entre différentes échelles d'action. Au départ, le gestionnaire de captages base son programme sur des outils mis en œuvre à l'échelle du territoire de l'AAC uniquement. Les actions d'animation et d'accompagnement individuel sont destinées aux agriculteurs ayant des parcelles sur l'AAC et sont couplées à la mobilisation d'outils parcellaires (MAET initialement puis maitrise foncière avec baux environnementaux). Ces outils correspondent à ceux traditionnellement mobilisés dans les démarches de protection des captages prioritaires : ils ciblent la parcelle et se concentrent sur l'AAC.

Mais les actions déployées ont progressivement évolué pour renforcer les impacts du projet. En effet, la durabilité et la viabilité économique d'une conversion à l'AB ne sont possibles que si des débouchés existent et permettent la collecte, parfois aussi la transformation, puis la mise en marché des produits agricoles sur des marchés rémunérateurs pour les agriculteurs. Eau de Paris s'implique donc à partir de 2012 dans des actions de développement et de structuration de nouveaux débouchés. Celles-ci sont menées à l'échelle élargie d'un «territoire de développement » qui correspond à l'espace sur lequel les acteurs agricoles s'appuient pour mener des actions de développement de l'AB (Vincent, 2016). Le cofinancement d'investissements matériels (stockage et triage) pour la station de semences, pourtant située hors du territoire de l'AAC, illustre cette évolution $d u$ raisonnement stratégique $d u$ gestionnaire de captages. Les interactions avec les acteurs de l'AB locaux (groupement d'agriculteurs biologiques et opérateurs économiques de l'aval) l'ont amené à aborder l'enjeu du développement de l'AB dans toute sa complexité, depuis l'accompagnement d'un cheminement individuel (Bellon et Lamine, 2009) et collectif jusqu'aux dimensions relatives à la mise en marché des produits. Ce changement de stratégie conduit le gestionnaire de captages à dépasser les limites du territoire de l'AAC (illustration 5). 
Illustration 5 - Mobilisation d'une diversité d'outils d'action publique permettant de toucher différentes échelles et cibles

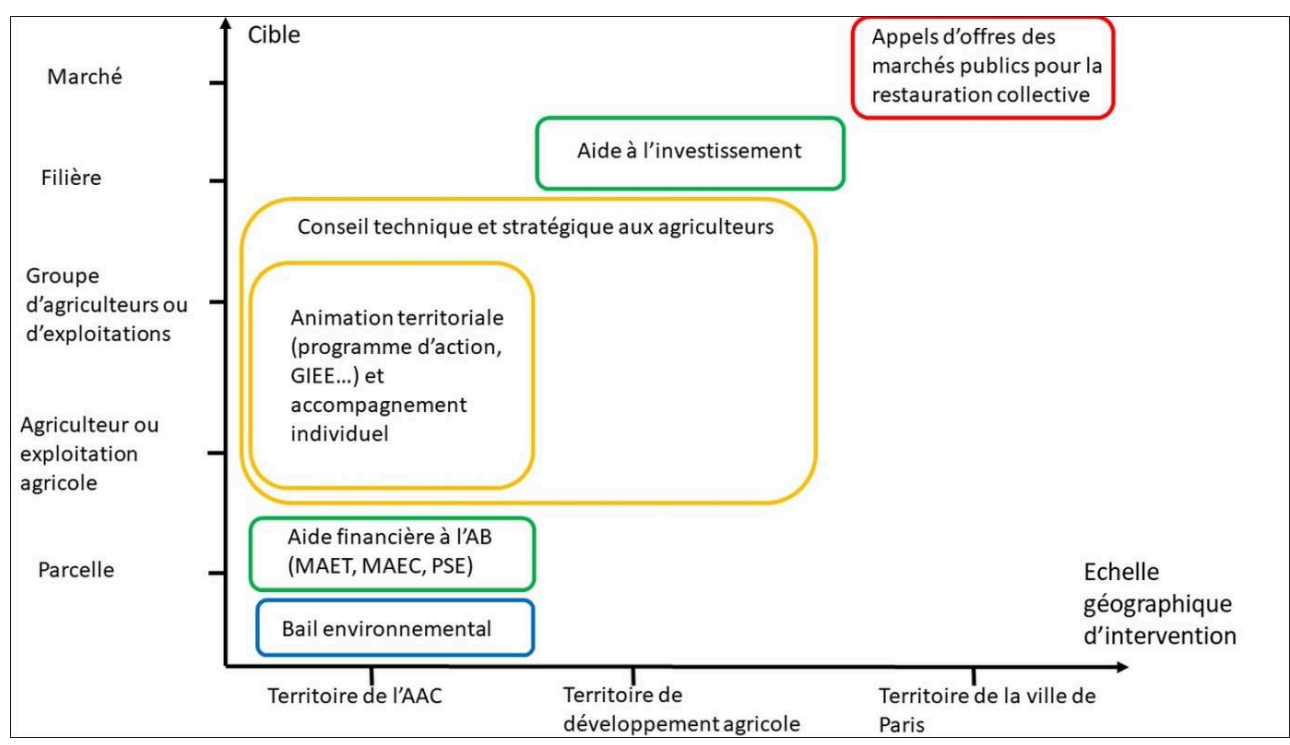

Outils fonciers en bleu

Aides financières directes en vert

Animation et accompagnement individuel en orange

Appels d'offres publics pour la restauration collective en rouge.

Réalisation : A. Vincent, P. Fleury.

Cette évolution du projet de reconquête de la qualité de l'eau sur la vallée de la Vanne témoigne d'une volonté forte du gestionnaire de captages de prendre en compte les besoins des acteurs locaux pour faire évoluer son projet et en améliorer la cohérence. Une fois les besoins identifiés, il recherche les outils pouvant être mobilisés au service du projet. En ce sens, le gestionnaire de captages joue un rôle central pour actionner divers outils d'action publique mais surtout pour penser leur articulation dans le temps et l'espace. Cette capacité à penser l'articulation et la complémentarité des outils et échelles d'action repose sur plusieurs facteurs. Tout d'abord, la régie peut s'appuyer sur sa solide équipe interne composée d'agents ayant une bonne connaissance des enjeux agricoles et agronomiques et du territoire et possédant des compétences en ingénierie de projets lui permettant de repérer les outils d'action publique mobilisables et de construire un projet complexe. La relative continuité et stabilité des agents en poste est sans doute également un facteur de réussite. Même s'il y a eu des changements d'animateurs territoriaux, le poste de responsable du service protection de la ressource est occupé par la même personne depuis le lancement du programme d'actions. Enfin les agents ont su nouer des partenariats stratégiques avec les acteurs agricoles du territoire les plus engagés sur l'AB (acteurs des filières notamment).

\section{Connecter les politiques de l'eau et de l'alimentation}

Sur la vallée de la Vanne, le gestionnaire de captages s'est donc rapidement engagé sur les questions de structuration de nouveaux débouchés en travaillant avec les coopératives biologiques locales qui disposent d'une expertise reconnue sur les attentes et l'évolution du marché. Les productions valorisées par leur intermédiaire sont vendues sur les marchés biologiques régional, national voire international (Allemagne notamment). Si ces débouchés déjà structurés existent, les agriculteurs à l'origine de 
l'association Terres du Pays d'Othe souhaitaient développer des marchés plus locaux et surtout assurer eux-mêmes la mise en marché de leurs produits. Grâce au Plan Alimentation Durable 2015-2020 de la ville de Paris affichant un objectif d'accroissement de l'approvisionnement en produits biologiques, l'idée d'introduire des produits biologiques issus de la vallée de la Vanne dans la restauration collective publique de la capitale a été remise à l'ordre du jour. Les premières livraisons ont été concrétisées fin 2017 par une vente de gré à gré.

Pour pérenniser le marché, il faut ensuite se conformer aux exigences du code des marchés publics. Pour les agriculteurs, cela requiert une organisation collective afin que la livraison et la facturation soient centralisées, un seul fournisseur étant retenu par lot. Pour la caisse des cantines des arrondissements intéressés, il s'agit de rédiger le marché public de telle sorte qu'il soit possible à un opérateur économique de petite taille d'y répondre voire qu'un lien soit mentionné entre la fourniture d'un produit alimentaire et la production d'une eau potable de qualité. L'association Terres du Pays d'Othe a remporté le premier marché public en 2019 avec la caisse des cantines du 11 ème arrondissement. D'autres arrondissements travaillent sur ce sujet également.

L'inscription dans le Plan Alimentation Durable d'une action intitulée "développer l'agriculture biologique sur les aires d'alimentation de captages d'Eau de Paris » témoigne de la volonté politique d'établir un lien entre politique de l'eau et politique de l'alimentation. Le descriptif de l'action précise qu'il s'agit de "poursuivre les actions qu'Eau de Paris a commencé à mettre en œuvre pour développer l'agriculture biologique sur les aires d'alimentation de captages (accompagnement des agriculteurs pour leur conversion au bio, acquisition foncière et mise en place de baux ruraux environnementaux)». La signature du premier marché en 2019 vient concrétiser cet objectif politique. Il s'agit effectivement d'une suite logique : proposer un débouché aux agriculteurs ayant accepté de faire évoluer leurs pratiques pour contribuer à la production d'une eau potable de qualité pour les Parisiens.

34 L'action d'Eau de Paris visant à engendrer des changements de fonds de l'agriculture n'est pas toujours bien accueillie. Certains agriculteurs acceptent mal que des changements importants leur soient demandés pour « que les Parisiens puissent boire de l'eau de qualité » comme le résume un agriculteur. Développer les débouchés dans la restauration collective publique de la capitale est une manière de formaliser le lien qui relie deux territoires certes disjoints (celui de la capitale et celui de l'AAC) mais dépendants; la dépendance étant à l'heure actuelle surtout celle de la capitale envers des territoires ruraux garants de son alimentation en eau potable. Pour la ville de Paris, il s'agit de marquer sa volonté de proposer une valorisation économique des produits issus de fermes engagées dans les pratiques plus vertueuses pour la qualité des ressources en eau qu'elle appelle de ses vœux et ainsi d'afficher une certaine cohérence politique.

Pour autant, les volumes vendus aux cantines parisiennes sont pour l'instant faibles : 1,5 tonne de lentilles en 2017, 7 tonnes de produits (lentilles, huiles...) en 2018, soit la valorisation des productions d'environ dix hectares de SAU. Le marché se développe mais il reste anecdotique si on le compare aux $23 \%$ de la SAU de l'AAC aujourd'hui consacrés à l'AB. Les volumes demandés par la restauration collective sont pour l'instant très faibles et ne concernent que certaines des productions de l'assolement (lentilles, tournesol, pois chiches). D'autres débouchés doivent donc être créés pour valoriser les autres productions. De surcroit, répondre à des marchés publics ciblant 
des produits issus de l'AAC nécessite que les opérateurs chargés de la collecte et de la mise en marché isolent les lots issus des exploitations de l'AAC. Cette séparation est pour l'heure trop contraignante et coûteuse au regard des très faibles volumes concernés et du dimensionnement des infrastructures de stockage des opérateurs assurant la collecte sur la zone. C'est pourquoi seuls deux ou trois producteurs engagés dans des logiques individuelles de commercialisation se sont lancés dans la réponse aux appels d'offres de la ville de Paris.

La restauration collective est un débouché qui ne permet donc pas d'assurer, à ce stade, la viabilité du projet de reconquête de la qualité de l'eau. Sur la vallée de la Vanne, Eau de Paris s'est très tôt mis en lien avec les coopératives assurant la collecte et la mise en marché des produits biologiques sur des circuits certes "longs » mais permettant de valoriser des volumes importants et la diversité des productions de la rotation. Les bons résultats, en termes de surfaces engagées, du projet de développement de l'AB sur la vallée de la Vanne sont multifactoriels mais tiennent notamment au fait que le gestionnaire de l'eau a su articuler trois échelles d'intervention (illustration 5): le territoire de l'AAC, le territoire de développement agricole, qui est celui à l'échelle duquel travaillent les opérateurs de la collecte et la mise en marché des productions biologiques et celui de la ville de Paris pour le développement d'un lien à l'alimentation.

L'idée de connecter politiques de l'eau et de l'alimentation pour assurer l'alimentation des Parisiens à partir des productions agricoles issues des AAC approvisionnant Paris en eau potable reste néanmoins séduisante. Des travaux de modélisation ont ainsi montré qu'il est techniquement possible de concevoir à l'échelle du bassin de la Seine un système agricole basé sur l'AB qui permettrait de nourrir la population francilienne tout en assurant la production d'une eau potable de qualité (Billen et al., 2011). Cette reconnexion entre les politiques de l'eau et de l'alimentation est importante même si elle n'est pour l'heure pas suffisamment structurée pour assurer un débouché à l'ensemble de l'agriculture d'une AAC. Ceci nécessiterait des changements importants pour les agriculteurs (adaptation des assolements et rotations pour implanter préférentiellement des cultures valorisables en restauration collective) et les opérateurs de la collecte et la mise en marché (isolement de lots par exemple). L'implication de transformateurs serait également requise pour tous les produits nécessitant une première transformation.

\section{Conclusion : pertinence d'une stratégie fondée uniquement sur l'AB}

Le cas emblématique du programme de reconquête de la qualité de l'eau sur la vallée de la Vanne se caractérise par un engagement très fort du gestionnaire de captages et par une volonté claire et assumée de privilégier l'AB. Le passage, entre 2008 et 2020, de 2 à $23 \%$ de la SAU en $\mathrm{AB}$ sur l'AAC est une progression bien supérieure à celle rencontrée à l'échelle nationale, passée elle de 2 à $8,5 \%$ de la SAU entre 2008 et 2019. Cette progression des surfaces en $A B$ résulte de conversions à l'AB (opérées par des agriculteurs qui conduisaient jusqu'alors leur exploitation en agriculture conventionnelle). Derrière cet indicateur quantitatif c'est une véritable transformation de la vision de l'AB en particulier dans sa capacité à contribuer positivement à l'avenir de l'agriculture du territoire que nous avons observé dans nos entretiens. De ce double 
point de vue, le programme peut être considéré comme une réussite. Il témoigne qu'il est possible d'enclencher et d'accompagner une dynamique territoriale de développement de l'AB à l'échelle d'une AAC en plaçant celle-ci au cœur du projet de reconquête de la qualité de l'eau et en élaborant un programme d'action tenant compte des spécificités locales et impliquant des acteurs partageant cet objectif.

Pourtant, au démarrage du projet, la vallée de la Vanne n'apparaissait pas nécessairement comme une zone agricole à fort potentiel de développement de l'AB. L'AB y était très peu présente et à la différence de l'exemple bien connu et souvent mis en avant de Munich (Barataud et al., 2014; Grolleau et McCann, 2012) où les systèmes agricoles étaient extensifs et très herbagers, la majorité des exploitations de la vallée de la Vanne sont des exploitations spécialisées en grandes cultures. Au-delà de l'articulation de différentes échelles d'intervention, cette réussite est due à plusieurs facteurs clefs, notamment à la volonté politique affirmée des élus d'Eau de Paris, à la mobilisation d'un large panel d'outils d'action publique et à leur maintien dans le temps.

Le choix fait par Eau de Paris de baser son projet de reconquête de la qualité de l'eau sur le seul développement de l'AB est un choix très rare. Il peut être « impopulaire » et fait courir le risque de déclencher des protestations fortes de la part des acteurs agricoles. Ainsi, la chambre d'agriculture de l'Yonne n'a pas souhaité être associée au projet pour montrer son désaccord. Eau de Paris a d'ailleurs fait des choix différents sur ses autres $A A C$ où l'AB n'est qu'une des options proposées aux agriculteurs; un accompagnement pour la réduction d'intrants en agriculture conventionnelle y étant également en place (Resch, 2020). Pour autant, les configurations locales ne sont pas figées. Elles peuvent évoluer rapidement par exemple lorsque les gestionnaires de l'eau et les collectivités gestionnaires de captages affirment leur volonté politique de développer l'AB et s'entourent d'agents ayant des compétences sur cette thématique et en ingénierie de projet pour susciter des dynamiques.

\section{BIBLIOGRAPHIE}

Allaire G., Cahuzac E., Maigné E., Poméon T., Simioni M., 2013. Réflexion à partir d'une analyse spatiale sur les politiques de soutien et la diffusion de l'agriculture biologique. Innovations agronomiques, $\mathrm{n}^{\circ} 32$, p. 227-241.

Barataud F., Aubry C., Wezel A., Mundler P., 2014. Management of drinking water catchment areas in cooperation with agriculture and the specific role of organic farming. Experiences from Germany and France. Land use policy, ${ }^{\circ}$ 36, p. 585-594.

Barataud F., Durpoix A., Mignolet C., 2014. Broad analysis of French priority catchment areas: A step toward adaption of the Water Framework Directive? Land use policy, vol. 36, p. 427-440.

Barraqué B., Viavattene C., 2009. Eau des villes et Eau des champs. Economie rurale, vol. 310, n² p. 5-21. 
Bellon S., Lamine C., 2009. Conversion to organic farming: a multidimensional research object at the crossroads of agricultural and social sciences. A review. Agronomy for Sustainable Development, $\mathrm{n}^{\circ} 29$, p. 97-112.

Billen G., Garnier J., Thieu V., Silvestre M., Barles S., Chatzimpiros P., 2011. Localising the nitrogen imprint of the Paris food supply: the potential of organic farming and changes in human diet. Biogeosciences Discussions, ${ }^{\circ}$ 8, p. 10979-11002.

Epices, ADE, 2017. Evaluation ex-post du programme de développement rural hexagonal (PDRH), programmation FEADER 2007/2013, Tome 2,174 p.

Epices, 2016. Analyse comparative des actions et dynamiques territoriales sur les sites " eau et bio ", Rapport de synthèse, $29 \mathrm{p}$.

Epices et AScA, 2020. Etude de synthèse bibliographique sur les actions destinées à lutter contre les pollutions diffuses agricoles des captages d'eau potables. Synthèse des résultats et propositions, $47 \mathrm{p}$.

Fleury P. 2011. Agriculture biologique et environnement : des enjeux convergents. Éducagri éditions ACTA Publications, $270 \mathrm{p}$.

Grolleau G., McCann L. M. J., 2012. Designing watershed programs to pay farmers for water quality services: Case studies of Munich and New York City. Ecological Economics, n 76, p. 87-94.

Lamine C., Meynard J.-M., Perrot N., Bellon S., 2009. Analyse des formes de transition vers des agricultures plus écologiques: les cas de l'agriculture biologique et de la protection intégrée. Innovations Agronomiques, n 4, p. 483-493.

Musselin C., 2005. Sociologie de l'action organisée et analyse des politiques publiques : deux approches pour un même objet ? Revue française de science politique, vol. 55, $\mathrm{n}^{\circ} 1$, p. 51-71. URL : https://hal-sciencespo.archives-ouvertes.fr/hal-01561196/document

Petit C., Vincent A., Fleury P., Durpoix A., Barataud, F., 2016. Protecting Water from Agricultural Diffuse Pollutions: Between Action Territories and Hydrogeological Demarcation Water Resources Management, vol. 30, ${ }^{\circ}$, p. 295-313

Resch M., 2020. Les territoires d'approvisionnement en eau potable de Paris. Du transfert à la préservation des ressources. Thèse de doctorat en Géographie et Aménagement. Université de Paris, 310 p.

Thieu V., Billen G., Garnier J., Benoît M., 2011. Nitrogen cycling in a hypothetical scenario of generalised organic agriculture in the Seine, Somme and Scheldt watersheds. Regional Environmental Change, vol. 11, n² 2, p. 359-370.

Vincent A., Fleury P., 2015. Development of organic farming for the protection of water quality: Local projects in France and their policy implications. Land use Policy, vol. 43, p. 197-206.

Vincent A., 2016. L'agriculture biologique comme réponse à la pollution de l'eau. Apports de la géographie pour comprendre les dynamiques en cours. Thèse de doctorat en Géographie, Aménagement, Urbanisme. Université Lumière Lyon 2, 401 p.

Zakeossian M., 2011. Protection des eaux souterraines : exemples d'actions engagées dans le domaine agricole par Eau de Paris. Responsabilité \& Environnement, vol. 63, p. 80-85.

\section{NOTES}

1. La loi stipule que «sur les périmètres de captage d'eau potable, la priorité sera donnée aux surfaces d'agriculture biologique et d'agriculture faiblement utilisatrice d'intrants afin de préserver la ressource en eau [...] » (Article 27 de la Loi). 
2. Le Groupement d'Intérêt Economique et Environnemental est une reconnaissance accordée par l'Etat à des collectifs d'agriculteurs engagés dans la modification de leurs pratiques pour une prise en compte accrue des enjeux environnementaux, économiques et sociaux (ou la consolidation de pratiques vertueuses).

3. En 2020 , le GIEE compte 27 adhérents.

\section{RÉSUMÉS}

L'agriculture biologique $(\mathrm{AB})$ est considérée comme une solution pertinente aux problèmes de pollution des ressources en eau. Dans cet article, nous nous appuyons sur le projet mené par la ville de Paris dans la vallée de la Vanne pour analyser comment une agglomération peut développer un projet de développement de l'AB à l'échelle d'une aire d'alimentation de captages et engager un changement de fonds de l'agriculture afin de pérenniser son approvisionnement en eau potable. Nous retraçons la trajectoire de ce projet et montrons que différents outils et échelles d'action ont été mobilisés pour mettre en lien l'enjeu eau potable avec d'autres comme celui de l'alimentation via l'approvisionnement de cantines en produits biologiques.

Organic farming (OF) is seen as a relevant solution to tackle non-point water pollutions. In this paper, we analyse the initiative carried out by the city of Paris to lead to a place-based development of OF in one of its water catchment area (vallée de la Vanne, Yonne). Paris' water authorities have set up a local organic action plan. We present the project's main stages. We show that different policy tools and levels of action have been implemented in order to connect the drinking water issue with others such as economic development and food policies (via green public procurements). A scheme to support OF through payments for environmental services has also been established.

\section{INDEX}

Mots-clés : agriculture biologique, aire d'alimentation de captages, alimentation, restauration collective, paiement pour services environnementaux

Keywords : organic farming, water catchment area, water policy, food policy, payment for environmental services

Thèmes : Sur le Champ

\section{AUTEURS}

\section{AUDREY VINCENT}

Audrey Vincent, avincent@isara.fr, est enseignante-chercheure, à l'ISARA, Laboratoire d'études rurales, Université Lyon 2. Elle a récemment publié :

- Berthet A., Vincent A., Fleury F., 2021. Water quality issues and agriculture: An international review of innovative policy schemes. Land Use policy [En ligne], vol. 109.

- Wezel A., Vincent A., Nitsch H., et al., 2018. Farmers' perceptions, preferences, and propositions 
for result-oriented measures in mountain farming. Land Use Policy, $\mathrm{n}^{\circ}$ 70, p. 117-127.

- Petit, C., Vincent A., Fleury P., Durpoix A., Barataud F., 2016. Protecting Water from Agricultural Diffuse Pollutions: Between Action Territories and Hydrogeological Demarcation. Water Resources Management, vol. 30, n 1, p. 295-313.

\section{PHILIPPE FLEURY}

Philippe Fleury, pfleury@isara.fr, est enseignant-chercheur à l'ISARA, Laboratoire d'études rurales, Université Lyon 2. Il a récemment publié :

- Fleury P. 2021. Explorer, équiper et représenter grottes et réseaux. Techniques \& Culture [En ligne], Suppléments au n75. URL: http://journals.openedition.org/tc/15165

- Delclaux, J., Fleury, P., 2020. Medium-term evolution in French national newspaper coverage of the interrelations between biodiversity and agriculture. Conservation Science and Practice. DOI: https://doi.org/10.1111/csp2.140

- Delclaux, J., Fleury, P., 2020. Politique de conservation de la biodiversité et d'aménagement du territoire : état de l'art sur la mise en œuvre de la Trame verte et bleue en France. Cybergeo: European Journal of Geography [En ligne], document 961. URL: http://journals.openedition.org/ cybergeo/35801 - DOI: https://doi.org/10.4000/cybergeo.35801 